\title{
Confluence without Termination via Parallel Critical Pairs
}

\author{
Bernhard Gramlich* \\ Fachbereich Informatik, Universität Kaiserslautern \\ Postfach 3049, 67653 Kaiserslautern, Germany \\ gramlich@informatik.uni-kl.de
}

SEKI-Report SR-95-13 (SFB)

\begin{abstract}
We present a new criterion for confluence of (possibly) non-terminating leftlinear term rewriting systems. The criterion is based on certain strong joinability properties of parallel critical pairs. We show how this criterion relates to other well-known results, consider some special cases and discuss some possible extensions.
\end{abstract}

\section{Introduction and Overview}

Computation formalisms which are based on rewriting systems heavily rely on the fundamental properties of termination and confluence. For terminating and confluent systems normal forms exist and are unique, irrespective of the computation (rewriting) strategy. For non-terminating but confluent systems, normal forms need not exist, however, if a normal form exists, it is still unique. More generally, any (possibly infinite) diverging computations can be joined again. In some cases, non-termination is inherently unavoidable, in other cases it may be very difficult to verify this property. Hence the problem of proving confluence (with or without termination) is of fundamental importance.

For abstract reduction (or abstract rewriting) systems (ARSs for short) it is well-known that, under termination, confluence is equivalent to local confluence, via Newman's Lemma. For proving confluence of non-terminating ARSs, however, one usually needs much stronger local confluence properties. A very interesting unifying framework, based on so-called decreasing diagrams, for localizing confluence proofs (even without

\footnotetext{
${ }^{*}$ This research was supported by the 'Deutsche Forschungsgemeinschaft, SFB 314 (D4-Projekt)'.
} 
termination) in the general setting of labelled abstract rewriting systems has recently been developed by van Oostrom ([Oos94a]).

For term rewriting systems (TRSs for short), which are ARSs with some additional structure, local confluence can be characterized by confluence of critical pairs as expressed by the well-known Critical Pair Lemma. Hence, for (finite) terminating TRSs, this critical pair test yields decidability of confluence.

For non-terminating TRSs, however, the situation is much more difficult again. Even the absence of critical pairs does not guarantee confluence, as there exist non-terminating, non-overlapping TRSs which are (locally confluent but) not confluent (cf. e.g. [Hue80]). These counterexamples must necessarily be non-left-linear. ${ }^{1}$ In fact, TRSs which are left-linear and non-overlapping, i.e., orthogonal, are confluent (cf. e.g. [Ros73]). This fundamentally important positive result has been considerably generalized by Huet $([$ Hue 80$])$ and further by Toyama ([Toy 88]) by allowing critical pairs, but imposing certain strong joinability properties on them (cf. Theorems 3.2, 3.5 and 3.7 below). The first result, Theorem 3.2, however, has the severe drawback that it additionally requires right-linearity, a 'rather unnatural condition' as pointed out in [Hue80]. Theorems 3.5 and 3.7 of Huet/Toyama are particularly interesting, since they do not require right-linearity. They are proved by showing strong confluence of parallel reduction, making essential use of the above mentioned particular joinability properties of (ordinary) critical pairs.

Our main new (and quite natural) idea now is that in order to ensure strong confluence of parallel reduction, one may also define and investigate the corresponding notion of parallel critical pairs. This new concept indeed turns out to be very useful, since we are able to state and prove a new sufficient condition for strong confluence of parallel reduction (cf. Theorem 4.11) which is based on certain joinability properties for parallel critical pairs (cf. Definition 4.8).

Actually, the idea behind parallel critical pairs is not completely new. Implicitly, parallel critical pairs - or, more precisely, parallel critical peaks - are at the heart of so-called critical pair criteria for completion of terminating TRSs (cf. [WB86], [Küc85], [KMN88], [BD88]), where certain (ordinary) critical pairs can be ignored during completion since they are redundant.

The rest of the paper is structured as follows. After introducing the necessary terminology, we present in Section 3 the known related results, give an example where none of the known confluence criteria applies, and motivate the introduction of parallel critical pairs. The main result of the paper, Theorem 4.11, is proved in Section 4. Its relation to the previous results, various illuminating examples and other related work are discussed in Section 5. Finally we conclude by discussing directions for further extending and generalizing our approach.

\footnotetext{
${ }^{1}$ Some interesting efforts for isolating the essence of the source of non-confluence in this case are described e.g. in [OT94], [TO95]. A decidable sufficient (syntactic) condition for confluence of nonleft-linear, non-terminating TRSs is given in [TO95].
} 


\section{Preliminaries}

We assume familiarity with the basic theory of abstract reduction systems as well as of the special case of term rewriting systems. For comprehensive surveys see e.g. [Klo92], [DJ90], [AM90], [Pla94]. Furthermore we also use some basic facts about unification theory (cf. e.g. [BS94] for a recent survey).

\subsection{Abstract Reduction Systems}

An abstract reduction system (ARS) is a pair $\mathcal{A}=\langle A, \rightarrow\rangle$ consisting of a (base) set $A$ and a binary relation $\rightarrow \subseteq A \times A$ also called (abstract) reduction or (abstract) rewrite relation. We use the standard notations $\rightarrow^{+}, \rightarrow^{*}, \rightarrow^{=}\left(\right.$or $\left.\rightarrow^{\leq 1}\right)$ for the transitive, transitive-reflexive and reflexive closure, respectively, of $\rightarrow$. The notations for the inverse relations are obtained by 'mirroring', e.g., we use $\leftarrow$ for the inverse of $\rightarrow$. Relation composition is denoted by $\circ$. Two elements $a, b \in A$ are said to be joinable (denoted by $a \downarrow b$ ) if there exists $c \in A: a \rightarrow^{*} c^{*} \leftarrow b$. If $a \rightarrow^{*} b$, we call $b$ a reduct of a. The identity relation on $A$ is given by $i d_{A}=\{(a, a) \mid a \in A\}$.

\section{Definition 2.1 (confluence properties)}

Let $\mathcal{A}=\langle A, \rightarrow\rangle$ be an ARS. Then $\mathcal{A}($ or $\rightarrow)$

- is confluent (CONF) if ${ }^{*} \leftarrow \circ \rightarrow^{*} \subseteq \rightarrow^{*} \circ^{*} \leftarrow$.

- is Church-Rosser (CR) if $\leftrightarrow^{*} \subseteq \rightarrow^{*} 0^{*} \leftarrow$.

- is locally confluent or weakly Church-Rosser (WCR) if $\leftarrow \circ \rightarrow \subseteq \rightarrow^{*} \circ{ }^{*} \leftarrow$.

- is strongly confluent (SCR) if $\leftarrow \circ \rightarrow \subseteq \rightarrow^{*} \circ=\leftarrow$.

- is subcommutative ( $\mathrm{WCR}^{\leq 1}$ ) if $\leftarrow \circ \rightarrow \subseteq \rightarrow=\circ \leftarrow$.

- is uniformly confluent $\left(\mathrm{WCR}^{1}\right)$ if $\leftarrow \circ \rightarrow \subseteq \mathrm{id}_{A} \cup(\rightarrow \circ \leftarrow)$.

- has the diamond property $(\diamond)$ if $\leftarrow \circ \rightarrow \subseteq \rightarrow \circ \leftarrow$.

Note that if the reduction relation $\rightarrow$ of $\mathcal{A}=\langle A, \rightarrow\rangle$ is reflexive, then the diamond property, subcommutativity and uniform confluence (of $\mathcal{A}$ ) are equivalent.

Confluence of an ARS $\mathcal{A}=\langle A, \rightarrow\rangle$ can be characterized in various equivalent ways. For instance, we have:

$$
\mathrm{CR}(\rightarrow) \Longleftrightarrow \mathrm{CONF}(\rightarrow) \Longleftrightarrow \mathrm{CR}\left(\rightarrow^{*}\right) \Longleftrightarrow \leftarrow \circ \rightarrow^{*} \subseteq \rightarrow^{*} \circ{ }^{*} \leftarrow
$$

Justified by the equivalence of the first two properties above and following a widespread convention we shall subsequently denote the confluence property by CR.

We note that due to the equivalence $\mathrm{CR}(\rightarrow) \Longleftrightarrow \mathrm{CR}\left(\rightarrow^{*}\right)$ (by idempotence of $*^{*}$ ), for proving confluence of $\rightarrow$ it suffices to prove confluence of $\rightsquigarrow$ for some reduction relation $\rightsquigarrow$ with the same transitive-reflexive closure, i.e. with $\rightsquigarrow^{*}=\rightarrow^{*}$. 
The next well-known result summarizes the relationships between the various confluence properties introduced in Definition 2.1 above. It provides interesting sufficient criteria for proving confluence by strengthened versions of local confluence.

\section{Theorem 2.2 (confluence by strengthening local confluence)}

Let $\mathcal{A}=\langle A, \rightarrow\rangle$ be an ARS. Then the following implications hold:

$$
\diamond \Longrightarrow \mathrm{WCR}^{1} \Longrightarrow \mathrm{WCR}^{\leq 1} \Longrightarrow \mathrm{SCR} \Longrightarrow \mathrm{CR}
$$

Local confluence (WCR) does not imply confluence (CR) in general, but only under the additional assumption of termination. This fundamentally important result, known as Newman's Lemma, is at the heart of many confluence proofs in the literature.

Here, an ARS $\mathcal{A}=\langle A, \rightarrow\rangle$ (or $\rightarrow$ ) is said to be terminating if there exists no infinite reduction sequence $a_{0} \rightarrow a_{1} \rightarrow a_{2} \rightarrow \ldots$ (of elements of $A$ ). $\mathcal{A}($ or $\rightarrow$ ) is called complete (or convergent) if it is confluent and terminating.

Theorem 2.3 (Newman's Lemma, [New42])

A terminating ARS $\mathcal{A}=\langle A, \rightarrow\rangle$ is confluent if and only if it is locally confluent.

\section{$2.2 \quad$ Term Rewriting Systems}

A term rewriting system $\mathcal{R}$ (over some set $\mathcal{T}(\mathcal{F}, \mathcal{V}$ ) of terms) may be viewed as the ARS $\left\langle\mathcal{T}(\mathcal{F}, \mathcal{V}), \rightarrow_{\mathcal{R}}\right\rangle$ where $\rightarrow_{\mathcal{R}}$ or simply $\rightarrow$ is the rewrite relation induced by the rewrite rules $l \rightarrow r \in \mathcal{R}(l, r \in \mathcal{T}(\mathcal{F}, \mathcal{V})$ with $\operatorname{Var}(r) \subseteq \operatorname{Var}(l))$. A term is linear if no variable occurs more than once in it. A rewrite rule $l \rightarrow r$ is said to be left-linear / right-linear if $l / r$ is linear. A TRS is left-linear / right-linear if all its rules are left-linear / right-linear.

For substitutions we use prefix notation, sometimes omitting parentheses, e.g., we write $\sigma(s)$ or $\sigma s$ to denote the result of applying the substitution $\sigma$ to the term $s$. The domain and the variable range of a substitution $\sigma$ are denoted by $\operatorname{Dom}(\sigma)$ (or $\operatorname{Dom} \sigma$ ) and $\mathcal{V} \operatorname{Ran}(\sigma)$ (or $\mathcal{V} \operatorname{Ran} \sigma$ ), respectively.

Positions (of terms) are also defined as usual, and denoted by $p, q$ etc.. They are ordered by $\leq$ as usual. The 'empty' root position is denoted by $\lambda$. Concatenation of positions is denoted by juxtaposition. If $p \leq q$ we say that $p$ is above $q$ (or $q$ is below $p$ ). For subtracting positions we use $\backslash$, i.e., we write $p \backslash q=q^{\prime}$ if $p=q q^{\prime}$. Two positions $p$ and $q$ are said to be parallel (or independent, disjoint), denoted by $p \| q$, if neither $p \leq q$ nor $q \leq p$. These notations extend in a straightforward way to sets of positions.

The set of positions of a term $s$ is denoted by $\operatorname{Pos}(s)$. The sets of variable positions and of non-variable, i.e., function symbol, positions of $s$ are denoted by $\mathcal{V} \operatorname{Pos}(s)$ and $\mathcal{F} \operatorname{Pos}(s)$, respectively. The subterm of $s$ at some position $p \in \operatorname{Pos}(s)$ is denoted by $s / p$. In order to indicate that $t$ is the subterm of $s$ at position $p \in \operatorname{Pos}(s)$, we also write $s=s[p=t]$. The result of replacing in $s$ the subterm at position $p \in \operatorname{Pos}(s)$ by $t$ is denoted by $s[p \leftarrow t]$. The result of (simultaneously) replacing in $s$ the subterms at some set $P=\left\{p_{1}, \ldots, p_{n}\right\} \subseteq \operatorname{Pos}(s)$ of parallel positions (of $s$ ) by $t_{1}, \ldots, t_{n}$, respectively, is 
denoted by $s\left[p_{1} \leftarrow t_{1}\right] \ldots\left[p_{n} \leftarrow t_{n}\right]$ (where the order of the replacement is irrelevant) or simply $s\left[p_{i} \leftarrow t_{i} \mid 1 \leq i \leq n\right]$. Similarly, $s\left[p_{i}=t_{i} \mid 1 \leq i \leq n\right]$ denotes $s$ indicating additionally that $s / p_{i}=t_{i}$ for all $i, 1 \leq i \leq n$.

If a term $s$ rewrites to $t$ at position $p \in \operatorname{Pos}(s)$ using rule $l \rightarrow r$ and substitution $\sigma$, i.e., $s / p=\sigma l, t=s[p \leftarrow \sigma r]$, this is indicated by using the notation $s \rightarrow_{p, \sigma, l \rightarrow r} t$ or, when ignoring the applied rule and used substitution but keeping the position of the contracted (i.e., replaced) redex (i.e., reducible expression), by $s \rightarrow_{p} t$. Furthermore, we write $s \rightarrow \geq p$ if $s$ reduces (in one step) to $t$ by contracting some redex (in $s$ ) below $p \in \operatorname{Pos}(s)$, and similarly for $s \rightarrow \leq p t$.

A unification problem is a (finite) set (or multiset) of equations to be solved. Here we use $\doteq$ in order distinguish e.g. the singleton unification problem $s \doteq t$ from the (syntactic) equality $s=t$ (cf. [BS94] for further details).

For testing local confluence of TRSs the following notion originating from [KB70] is fundamental.

\section{Definition 2.4 (critical pairs)}

Let $\mathcal{R}$ be a TRS and let $l \rightarrow r, l^{\prime} \rightarrow r^{\prime}$ be two rules of $\mathcal{R}$ that w.l.o.g. do not have any common variable. ${ }^{2}$ Suppose $l / p$, with $p \in \mathcal{F} \operatorname{Pos}(l)$, and $l^{\prime}$ are unifiable with mgu $\sigma$, where $\mathcal{V} \operatorname{Ran}(\sigma) \cap(\operatorname{Var}(l) \backslash \operatorname{Var}(l / p))=\emptyset .^{3}$ Then the pair of reducts $\left\langle(\sigma l)\left[p \leftarrow \sigma r^{\prime}\right], \sigma r\right\rangle$ is called a critical pair of $\mathcal{R}$, determined by overlapping $l^{\prime} \rightarrow r^{\prime}$ into $l \rightarrow r$ at position $p$. The divergence $(\sigma l)\left[p \leftarrow \sigma r^{\prime}\right]_{p} \leftarrow \sigma l \rightarrow_{\lambda} \sigma r$ is the corresponding critical peak. If $p=\lambda$, we speak of an outside critical peak (or critical overlay)and outside critical pair, respectively. Otherwise, i.e., if $p>\lambda$, we speak of an inside critical peak and inside critical pair, respectively (following [Toy88]). If the two rules are renamed versions of the same rule we do not consider the case $p=\lambda$ (which gives only rise to improper divergences). The set of all critical pairs between rules of $\mathcal{R}$ is denoted by $\operatorname{CP}(\mathcal{R})$. If $\operatorname{CP}(\mathcal{R})=\emptyset, \mathcal{R}$ is said to be non-overlapping. It is called orthogonal if it is nonoverlapping and left-linear.

Observe the asymmetry in the definition of critical pairs. This entails in particular, that for a critical overlay $t_{1} \lambda \leftarrow s \rightarrow_{\lambda} t_{2}$ we always get two corresponding critical pairs, namely $\left\langle t_{1}, t_{2}\right\rangle$ and $\left\langle t_{2}, t_{1}\right\rangle$. Moreover we note, that a critical pair may correspond to (i.e., be obtained from) several distinct critical peaks (if taking into account the position of the inside rewrite step and the applied rules). ${ }^{4}$

Lemma 2.5 (Critical Pair Lemma, [Hue80])

A TRS is locally confluent if and only if all its critical pairs are joinable.

Combining Newman's Lemma and the Critical Pair Lemma yields the following fundamental result.

\footnotetext{
${ }^{2}$ Note that considering renamed versions of the same rule of $\mathcal{R}$ is allowed here, too.

${ }^{3}$ This variable condition ensures that the mgu $\sigma$ does not introduce unnecessary identifications of variables in $l$. In case that the mgu of $t=l / p$ and $l^{\prime}$ is taken such that no new variables are introduced (which is always possible), this condition is vacuously satified.

${ }^{4}$ For the sake of readability we dispense here with a completely formal definition of critical peaks which is straightforward.
} 
Theorem 2.6 ([KB70])

A terminating TRS is confluent (hence complete) if and only if all its critical pairs are joinable.

\section{Known Results and Motivating Examples}

Without termination, showing confluence of a TRS is much more difficult. For leftlinear systems, a few results relying on strong confluence properties are known.

Definition 3.1 (strongly closed, [Hue80])

A TRS $\mathcal{R}$ is called strongly closed, which we denote by $\operatorname{SCCP}(\mathcal{R}),{ }^{5}$ if for every critical pair $\left\langle t_{1}, t_{2}\right\rangle$ there exist terms $t_{3}, t_{4}$ such that $t_{1} \rightarrow^{*} t_{3}=\leftarrow t_{2}$ and $t_{1} \rightarrow=t_{4}{ }^{*} \leftarrow t_{2}$.

Theorem 3.2 (strong confluence by strong closedness, [Hue80])

A left- and right-linear strongly closed TRS is strongly confluent, hence also confluent.

Proof: The proof in [Hue80] is by a complete case analysis for one-step divergences of the form $t_{1} \leftarrow s \rightarrow t_{2}$ exploiting strong closedness for the case that $t_{1} \leftarrow s \rightarrow t_{2}$ corresponds to an instance of a critical peak (note that right-linearity is needed for the case of a variable overlap).

The following very nice counterexample shows that right-linearity cannot be dropped in Theorem 3.2.

Example 3.3 (due to J.-J. Lévy as cited in [Hue80])

The TRS

$$
\mathcal{R}=\left\{\begin{aligned}
f(a, a) & \rightarrow g(b, b) \\
a & \rightarrow a^{\prime} \\
f\left(a^{\prime}, x\right) & \rightarrow f(x, x) \\
f\left(x, a^{\prime}\right) & \rightarrow f(x, x) \\
g(b, b) & \rightarrow f(a, a) \\
b & \rightarrow b^{\prime} \\
g\left(b^{\prime} x\right) & \rightarrow g(x, x) \\
g\left(x, b^{\prime}\right) & \rightarrow g(x, x)
\end{aligned}\right.
$$

is left-linear, non-right-linear and non-terminating. Moreover, it is easily verified that $\mathcal{R}$ is strongly closed, but non-confluent. In fact, we have e.g. $f\left(a^{\prime}, a^{\prime}\right){ }^{*} \leftarrow f(a, a) \rightarrow^{*}$ $g\left(b^{\prime}, b^{\prime}\right)$ but $f\left(a^{\prime}, a^{\prime}\right)$ and $g\left(b^{\prime}, b^{\prime}\right)$ do not have a common reduct (cf. Figure 1$)$.

For left-linear, but not necessarily right-linear TRSs one may use the following approach of Huet ([Hue 80$])$. Let $\mathcal{R}$ be a TRS. Then the parallel reduction relation (induced by $\mathcal{R}), \|_{\mathcal{R}}$ or simply $\nVdash$, is defined as the smallest reflexive relation containing $\rightarrow$ and verifying

$$
s_{1} \longrightarrow t_{1} \wedge \ldots s_{n} \longrightarrow t_{n} \Longrightarrow f\left(s_{1}, \ldots, s_{n}\right) \longrightarrow f\left(t_{1}, \ldots, t_{n}\right)
$$

\footnotetext{
${ }^{5}$ This is to abbreviate 'strongly closed critical pairs'.
} 


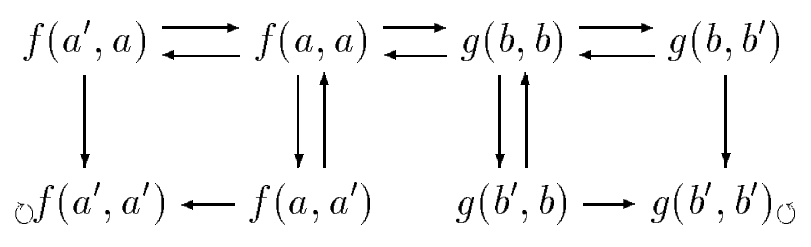

Figure 1: (partial) reduction graph of Example 3.3

for all $f \in \mathcal{F}$ (n-ary). If $s$ reduces to $t$ by a parallel step contracting the redexes in $s$ at some set $P=\left\{p_{1}, \ldots, p_{k}\right\}$ of parallel positions from $P$, this is also denoted by $s-{ }_{P} t$. Subsequently, we shall tacitly make use of some basic properties of parallel positions and parallel reduction, in particular the following ones:

- If $P_{1}, P_{2} \subseteq \operatorname{Pos}(s)$ are disjoint sets of positions of $s$ such that all positions in $P_{1} \uplus P_{2}$ are mutually disjoint, $t_{p} \rightarrow_{\lambda} t_{p}^{\prime}$ for all $p \in P_{1} \uplus P_{2}$, and $s=s[p=$ $\left.t_{p} \mid p \in P_{1}\right] \longrightarrow_{P_{1}} s\left[p \leftarrow t_{p}^{\prime} \mid p \in P_{1}\right]=s_{1}, s=s\left[p=t_{p} \mid p \in P_{2}\right] \longrightarrow_{P_{2}} s\left[p \leftarrow t_{p}^{\prime} \mid p \in\right.$ $\left.P_{2}\right]=s_{2}$, then, defining $s_{3}=s\left[p \leftarrow t_{p}^{\prime} \mid p \in P_{1} \uplus P_{2}\right]$, we get $s \mathbb{H}_{P_{1}} s_{1} \longrightarrow{ }_{P_{2}} s_{3}$, $s \longrightarrow{ }_{P_{2}} s_{2} \longrightarrow{ }_{P_{1}} s_{3}$, and $s \longrightarrow{ }_{P_{1} \cup P_{2}} s_{3}$ (by commutation of the two independent parallel steps, and by combining both into one parallel step).

- If $p \leq q$ and $p^{\prime} \| p$, then $p^{\prime} \| q$.

- If $P$ is a set of parallel positions of $s, q \in P o s(s)$ with $q \leq p$ for all $p \in P$, and $s, t_{1}$, $t_{2}$ coincide except for the respective subterms at position $q$, then $t_{1} P^{4} \| s \rightarrow_{q} t_{2}$ and $t_{1} / q Q \nVdash s / q \rightarrow_{\lambda} t_{2} / q$, where $Q=\{p \backslash q \mid p \in P\}$, are equivalent. In other words, the relevant part of a divergence $t_{1} P \mathbb{H} s \rightarrow_{q} t_{2}$ (with $q \leq p$ for all $p \in P$ ) can be obtained by extraction, stripping off the common context.

Clearly, parallel and ordinary reduction are related as follows: $\rightarrow \subseteq \longrightarrow \subseteq \rightarrow^{*}$, hence also $\rightarrow^{*}=\mathbb{H}^{*}$. Thus, for showing confluence of $\rightarrow$, it suffices to prove (strong) confluence of $\longrightarrow$.

Definition 3.4 (parallel closed, [Hue80])

A TRS $\mathcal{R}$ is called parallel closed if for every critical pair $\left\langle t_{1}, t_{2}\right\rangle$ of $\mathcal{R}$ we have: $t_{1} \longrightarrow \rightarrow t_{2}$.

Theorem 3.5 (subcommutativity of parallel reduction by parallel closedness, [Hue80])

If $\mathcal{R}$ is a left-linear parallel closed TRS, then parallel reduction (in $\mathcal{R}$ ) is subcommutative (WCR $\leq 1(-)$ ), hence $\mathcal{R}$ is confluent.

Proof: The ingenious proof in [Hue 80$]$ proceeds by considering parallel one-step di-

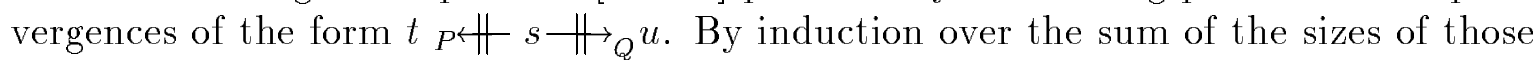
' $P$ - and $Q$-redexes' which are affected in both steps, and by case analysis according to 


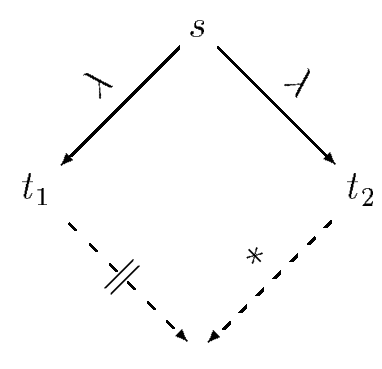

case (i))

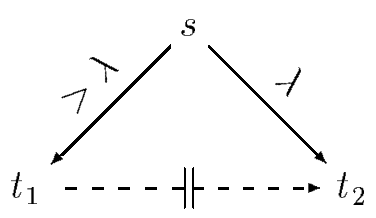

case (ii)

Figure 2: critical pair condition $\mathrm{CPC}_{\mathrm{HT}}$

the positions in $P, Q$, it is shown that $\longrightarrow$ is subcommutative ( $\mathrm{WCR}^{\leq 1}(\longrightarrow)$ ), hence confluent, which implies confluence of $\rightarrow$ since $\longrightarrow \rightarrow^{*}=\rightarrow^{*}$.

Toyama ([Toy88]) observed that, in Theorem 3.5 above, the condition that all critical pairs are parallel closed can be weakened without losing strong confluence of parallel reduction.

Definition 3.6 (critical pair condition of Huet/Toyama, [Toy88])

We say that a TRS $\mathcal{R}$ satisfies the critical pair condition $\mathrm{CPC}_{\mathrm{HT}},{ }^{6}$ denoted by $\mathrm{CPC}_{\mathrm{HT}}(\mathcal{R})$, if for every critical peak $D: t_{1} p^{\leftarrow s} \rightarrow_{\lambda} t_{2}$ of $\mathcal{R}$ (hence: $\left\langle t_{1}, t_{2}\right\rangle \in \mathrm{CP}(\mathcal{R})$ ) we have:

(i) If $p=\lambda$, i.e., if $D$ is an outside critical peak, then there exists a term $t_{3}$ such that $t_{1}-\| t_{3} * t_{2} \cdot{ }^{7}$

(ii) If $p>\lambda$, i.e., if $D$ is an inside critical peak, then $t_{1}-t_{2}$.

The conditions (i)-(ii) of Definition 3.6 are depicted in Figure 2 (dashed arrows in diagrams are existentially quantified).

\section{Theorem 3.7 (strong confluence of parallel reduction, [Toy88] ${ }^{8}$ )}

If $\mathcal{R}$ is a left-linear TRS satisfying the critical pair condition $\mathrm{CPC}_{\mathrm{HT}}$, then parallel reduction (in $\mathcal{R}$ ) is strongly confluent $(\operatorname{SCR}(\nVdash)$ ), hence $\mathcal{R}$ is confluent.

Proof: The proof in [Toy88] essentially proceeds as the one of [Hue80] for Theorem 3.5 above. One shows strong confluence of parallel reduction by considering parallel one-step divergences of the form $t_{P} \nVdash s \|_{Q} u$. The induction is over the sum of the

\footnotetext{
${ }^{6}$ Here, the subscript 'HT' is to abbreviate 'Huet/Toyama'.

${ }^{7}$ Note that due to the asymmetry in the definition of critical pairs / peaks this means that there must also exist a term $t_{4}$ with $t_{1} \rightarrow^{*} t_{4} \# t_{2}$ !

${ }^{8}$ In fact, the corresponding result in [Toy88], Corollary 3.2, p. 405, is obtained there by specializing a more general version, namely Theorem 3.1, p. 401, formulated in terms of a sufficient condition for commutativity of two left-linear TRSs.
} 
sizes of those ' $P$ - and $Q$-redexes' which are affected in both steps ignoring, however, overlaying redexes.

Before proceeding let us elaborate a bit on variations of critical pair conditions for ensuring confluence of left-linear TRSs. Huet's result above states that

(1) $s \nVdash t$ for every critical pair $\langle s, t\rangle$ of $\mathcal{R}$

suffices for ensuring confluence. Surprisingly, it still seems to be unknown whether any of the following conditions also suffices (see [DJK91], Problem 13 of J.-J. Lévy).

(2) $s \nrightarrow t$ or $t \nVdash s$, for every critical pair $\langle s, t\rangle$ of $\mathcal{R}$.

(3) $s \rightarrow^{=} t$ or $t \rightarrow=s$, for every critical pair $\langle s, t\rangle$ of $\mathcal{R}$.

(4) $t-\| s$ for every critical pair $\langle s, t\rangle$ of $\mathcal{R}$.

(5) $t \rightarrow=s$ for every critical pair $\langle s, t\rangle$ of $\mathcal{R}$.

Clearly, the following implications hold between these conditions: $(5) \Longrightarrow(3) \Longrightarrow(2)$, $(5) \Longrightarrow(4) \Longrightarrow(2)$. We remark that due to Theorem 3.2 potential counterexamples for (3) and (5) would have to be (besides left-linear) non-right-linear, non-terminating and non-orthogonal, hence overlapping. Unfortunately, our approach developed below does not solve any of these open problems.

For motivating our approach to showing confluence via strong confluence of parallel reduction let us now consider the following modified version of Lévy's counterexample 3.3 above.

\section{Example 3.8 (no known criterion applicable)}

The TRS

$$
\mathcal{R}=\left\{\begin{aligned}
f(a, a) & \rightarrow b \\
a & \rightarrow a^{\prime} \\
f\left(a^{\prime}, x\right) & \rightarrow f(x, x) \\
f\left(x, a^{\prime}\right) & \rightarrow f(x, x) \\
f\left(a^{\prime}, a^{\prime}\right) & \rightarrow b \\
b & \rightarrow f\left(a^{\prime}, a^{\prime}\right)
\end{aligned}\right.
$$

is left-linear, but not right-linear, and non-terminating (cf. Figure 3). Moreover, it is confluent which cannot be inferred from Theorem 3.7 since e.g. the inside critical peak $f\left(a^{\prime}, a\right) \leftarrow f(a, a) \rightarrow b$ is not parallel closed. Theorem 3.2 isn't applicable either, due to non-right-linearity of $\mathcal{R}$, though $\mathcal{R}$ is strongly closed. Nevertheless, parallel reduction (in $\mathcal{R}$ ) is strongly confluent (as will be shown). For instance, we observe that the divergence $f\left(a^{\prime}, a^{\prime}\right) \nVdash f(a, a) \rightarrow b$ can be 'strongly closed' via $f\left(a^{\prime}, a^{\prime}\right) \rightarrow b$ (or $b \rightarrow$ $\left.f\left(a^{\prime}, a^{\prime}\right)\right)$. In Example 3.3 we had the divergence $f\left(a^{\prime}, a^{\prime}\right) \nVdash f(a, a) \rightarrow g(b, b)$ which could only be closed via $g(b, b) \rightarrow f(a, a) \longrightarrow f\left(a^{\prime}, a^{\prime}\right)$, but not 'strongly', i.e., there was no term $t$ with $f\left(a^{\prime}, a^{\prime}\right) \rightarrow^{*} t \nVdash g(b, b)$. Furthermore we note that in both examples in the parallel step $f(a, a) \longrightarrow f\left(a^{\prime}, a^{\prime}\right)$ two parallel (inner) redexes are contracted both of which are critical w.r.t. the other outside step. 


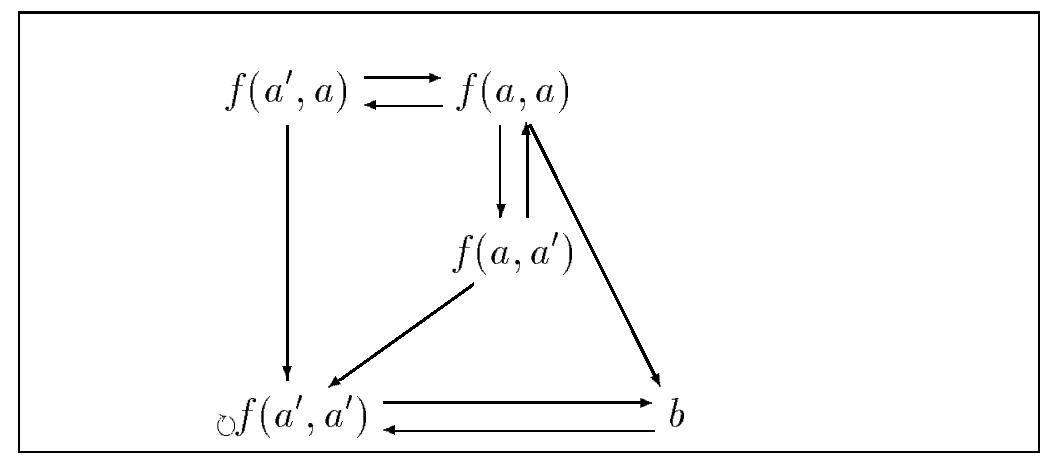

Figure 3: (partial) reduction graph for Example 3.8

Intuitively, the observations above suggest to analyse more carefully local divergences where in one parallel step several (parallel) redex positions are critical w.r.t. the other step. From a more abstract point of view this means to study the following questions:

(1) What are critical peaks / pairs for the parallel reduction relation?

(2) Which conditions on the corresponding critical peaks / pairs for parallel reduction guarantee strong confluence?

For (1) we shall introduce the notion of parallel critical peaks / pairs. In fact, Definition 4.1 below only accounts for critical divergences of the form $t_{1} \nVdash s \rightarrow_{\lambda} t_{2}$. However, this type of divergences (for parallel reduction) will turn out to be sufficient for characterizing strong confluence of $廿$ (cf. Lemma 4.9 below). Concerning (2) we shall present a parallel critical pair condition (cf. Definition 4.8) which indeed suffices for guaranteing strong confluence of $\longrightarrow$ (cf. Theorem 4.11).

\section{Main Result}

\section{Definition 4.1 (parallel critical pairs / peaks)}

Let $\mathcal{R}$ be a TRS and let $l \rightarrow r, l_{1} \rightarrow r_{1}, \ldots, l_{n} \rightarrow r_{n}$ be rules of $\mathcal{R}$ that w.l.o.g. do not have any common variable. Suppose $P=\left\{p_{1}, \ldots, p_{n}\right\}$ is a non-empty set of parallel non-variable positions of $l$. Suppose further that the unification problem $\left\{l_{i} \doteq l / p_{i} \mid 1 \leq\right.$ $i \leq n\}$ is solvable, let's say with mgu $\sigma$, where $\mathcal{V} \operatorname{Ran}(\sigma) \cap\left(\operatorname{Var}(l) \backslash\left(\bigcup_{i=1}^{n} \operatorname{Var}\left(l / p_{i}\right)\right)\right)=$ $\emptyset$. Then the pair of reducts $\left\langle(\sigma l)\left[p_{i} \leftarrow \sigma r_{i} \mid 1 \leq i \leq n\right], \sigma r\right\rangle$ is called a parallel critical pair of $\mathcal{R}$, determined by overlapping (in parallel) the rules $l_{i} \rightarrow r_{i}$ into $l \rightarrow r$ at the (parallel) positions $p_{i}$ (of $l$ ). The divergence $(\sigma l)\left[p_{i} \leftarrow \sigma r_{i} \mid 1 \leq i \leq n\right]_{P} \| \sigma l \rightarrow_{\lambda} \sigma r$ is the corresponding parallel critical peak. If $n=1$ and $l \rightarrow r, l_{1} \rightarrow r_{1}$ are two renamed versions of the same rules, we do not consider the case $p=\lambda$ (which gives only rise to improper divergences). The set of all parallel critical pairs between rules of $\mathcal{R}$ is denoted by $\operatorname{PCP}(\mathcal{R})$. A parallel critical peak (and its corresponding parallel critical 
pair) is said to be proper if the inside parallel step in it contracts more than one redex (with the notation from above: $n \geq 2$ ).

\section{Definition 4.2 (recursive computation of parallel critical pairs)}

Let $\mathcal{R}$ be a TRS. We recursively define the sets $\operatorname{PCP}_{k}(\mathcal{R})$ of parallel critical pairs of order $k$ by:

- $\operatorname{PCP}_{1}(\mathcal{R}):=\mathrm{CP}(\mathcal{R})$, i.e., the parallel critical pairs (and peaks, respectively) of order 1 are the ordinary critical pairs (and peaks, respectively) of $\mathcal{R}$.

- $\mathrm{PCP}_{k+1}(\mathcal{R})$ (for $k \geq 1$ ) is obtained from $\mathrm{PCP}_{k}(\mathcal{R})$ as follows: Let $t_{1}:=(\sigma l)\left[p_{i} \leftarrow\right.$ $\left.\sigma r_{i} \mid 1 \leq i \leq k\right]_{P} \| \sigma l \rightarrow_{\lambda} \sigma r=: t_{2}$, with $P=\left\{p_{1}, \ldots, p_{k}\right\} \subseteq \mathcal{F P o s}(l)$, be a parallel critical peak of order $k$, i.e., with $\left\langle t_{1}, t_{2}\right\rangle \in \operatorname{PCP}_{k}(\mathcal{R})$. Suppose $p_{k+1} \in$ $\mathcal{F} \operatorname{Pos}(l)$ with $p_{k+1} \| P$, i.e., $p_{k+1}$ is a non-variable position of $l$ which is parallel to $p_{1}, \ldots, p_{k}$.

Let $l_{k+1} \rightarrow r_{k+1}$ be a rule from $\mathcal{R}$ with fresh variables such that $\sigma\left(l / p_{k+1}\right)$ and $l_{k+1}$ are unifiable with mgu $\tau$, where $\mathcal{V} \operatorname{Ran}(\tau) \cap\left(\operatorname{Var}\left(t_{1}\right) \backslash\left(\bigcup_{i=1}^{k} t_{1} / p_{i}\right)\right)=\emptyset$. Then the divergence

$$
t_{1}^{\prime}:=(\tau \sigma l)\left[p_{i} \leftarrow \tau \sigma r_{i} \mid 1 \leq i \leq k\right]\left[p_{k+1} \leftarrow \tau r_{k+1}\right] Q \nVdash \tau \sigma l \rightarrow_{\lambda, \tau \sigma, l \rightarrow r} \tau \sigma r=: t_{2}^{\prime},
$$

where $Q=P \uplus\left\{p_{k+1}\right\}$ and where the parallel step contracts the redexes in $\tau \sigma l$ at the positions from $Q$ using the rules $l_{1} \rightarrow r_{1}, \ldots, l_{k+1}$, respectively, is a parallel critical peak of order $k+1$, and $\left\langle t_{1}^{\prime}, t_{2}^{\prime}\right\rangle$ the corresponding parallel critical pair of order $k+1$. Performing such an additional superposition for all parallel critical peaks of order $k$ in all possible ways, we get the set $\operatorname{PCP}_{k+1}(\mathcal{R})$ of all parallel critical pairs of order $k+1$ (with corresponding parallel critical peaks of order $k+1)$.

$$
\text { - } \operatorname{PCP}^{r e c}(\mathcal{R}):=\bigcup_{k \geq 1} \operatorname{PCP}_{k}(\mathcal{R}) \text {. }
$$

Remark 4.3 If in the above definition the size of the left hand sides of $\mathcal{R}$ is bounded, then there is a maximal $k$ for which $\mathrm{PCP}_{k}(\mathcal{R})$ may properly contribute to $\mathrm{PCP}^{r e c}(\mathcal{R})$. This is obvious, since for constructing a parallel critical peak of order $k$ we need at least $k$ function symbols occurring at $k$ parallel (non-variable) positions in some left hand side of $\mathcal{R}$. Hence, for any $m \geq \max _{l \rightarrow r \in \mathcal{R}} \|_{\mathcal{F}}(l)$, where the parallel function (symbol) width $\|_{\mathcal{F}}(s)$ of a term $s$ is defined by

$$
\|_{\mathcal{F}}(s)= \begin{cases}0 & \text { if } s \in \mathcal{V} \\ 1 & \text { if } s \in \mathcal{F} \\ \max \left\{1, \sum_{i=1}^{n} \|_{\mathcal{F}}\left(s_{i}\right)\right\} & \text { if } s=f\left(s_{1}, \ldots, s_{n}\right)\end{cases}
$$

we have $\operatorname{PCP}^{r e c}(\mathcal{R})=\bigcup_{k \geq 1}^{m} \operatorname{PCP}_{k}(\mathcal{R})$.

Lemma 4.4 For any TRS $\mathcal{R}$ the sets $\operatorname{PCP}(\mathcal{R})$ and $\operatorname{PCP}^{r e c}(\mathcal{R})$ coincide (modulo variable renamings). 
Proof: Straightforward, by standard rewriting (and unification) techniques. One only has to use the basic fact, that solving a given unification problem $E \cup\{s \doteq t\}$ can be recursively done by first solving $E$ yielding (in case of solvability) a most general unifier $\sigma$ (via a solved form of $E$ ), and then solving additionally $\sigma s \doteq \sigma t$ producing (in case of solvability) let's say the mgu $\psi$. Then, the composition of $\sigma$ and $\psi, \sigma \circ \psi$, is an mgu of the original unification problem $E \cup\{s \doteq t\}$.

In order to get some intuition for the introduced notion of parallel critical pairs / peaks and their computation let us give two simple examples.

Example 4.5 The TRS

$$
\mathcal{R}=\left\{\begin{aligned}
f(g(x), h(x, y)) & \rightarrow a \\
g(b) & \rightarrow c \\
h(x, d) & \rightarrow e
\end{aligned}\right.
$$

has two (parallel) critical peaks of order 1, namely $f(c, h(b, y)) 1_{1} \leftarrow f(g(b), h(b, y)) \rightarrow_{\lambda} a$ and $f(g(x), e){ }_{2} \leftarrow f(g(x), h(x, d)) \rightarrow_{\lambda} a$, obtained by overlapping the second and the third rule, respectively, into the first one. And there is one (proper) parallel critical peak of order 2, namely $f(c, e)\{1,2\} \mathbb{W} f(g(b), h(b, d)) \rightarrow_{\lambda} a$, obtained either directly by overlapping in parallel the last two rules into the first, or recursively from both critical peaks of order 1 by an additional superposition. Hence, we have $\operatorname{PCP}_{1}(\mathcal{R})=$ $\{\langle f(c, h(b, y)), a\rangle,\langle f(g(x), e), a\rangle\}, \mathrm{PCP}_{2}(\mathcal{R})=\{\langle f(c, e), a\rangle\}$, and $\operatorname{PCP}(\mathcal{R})=\operatorname{PCP}_{1}(\mathcal{R}) \cup$ $\mathrm{PCP}_{2}(\mathcal{R})$.

Example 4.6 The TRS

$$
\mathcal{R}=\left\{\begin{aligned}
f(g(x), h(x)) & \rightarrow a \\
g(b) & \rightarrow d \\
h(c) & \rightarrow d
\end{aligned}\right.
$$

has two (parallel) critical peaks of order 1 , namely $f(d, h(b)) 1_{1} \leftarrow f(g(b), h(b)) \rightarrow_{\lambda} a$ and $f(g(c), d){ }_{2} \leftarrow f(g(c), h(c)) \rightarrow_{\lambda} a$, obtained by overlapping the second and the third rule, respectively, into the first one. However, there is no (proper) parallel critical peak of order 2, since simultaneously unifying $g(x)$ with $g(b)$ and $h(x)$ with $h(c)$ obviously fails. Hence, we get $\operatorname{PCP}(\mathcal{R})=\operatorname{PCP}_{1}(\mathcal{R})=\{\langle f(d, h(b)), a\rangle,\langle f(g(c), d), a\rangle\}$.

Lemma 4.7 Let $\mathcal{R}$ be a TRS. Then any divergence $t_{1}^{\prime} \nVdash s^{\prime} \rightarrow_{\lambda} t_{2}^{\prime}$, with $s^{\prime} \rightarrow_{\lambda, \sigma, l \rightarrow r} t_{2}^{\prime}$ and $s^{\prime} \longrightarrow{ }_{P} t_{1}^{\prime}, P$ some set of $n$ parallel non-variable positions $p_{i}(1 \leq i \leq n)$ from $l$, is an instance of a parallel critical peak $t_{1} P \mathbb{H} \rightarrow_{\lambda} t_{2}$ of $\mathcal{R}$ (of order $n$ ).

Proof: Routine, by a straightforward peak analysis analogous to the situation for ordinary critical pairs (as it is used in the proof of the Critical Pair Lemma 2.5).

Next we introduce the central condition on parallel critical pairs that will enable us to prove strong confluence of parallel reduction.

\section{Definition 4.8 (parallel critical pair condition)}

We say that a TRS $\mathcal{R}$ satisfies the parallel critical pair condition $\mathrm{PCPC}(\mathrm{PCPC}(\mathcal{R})$ for short) if for every parallel critical peak $D: t_{1} P^{4} s \rightarrow_{\lambda} t_{2}$ of $\mathcal{R}$ the following holds: 


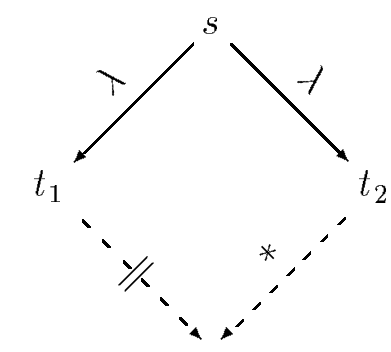

case (i)

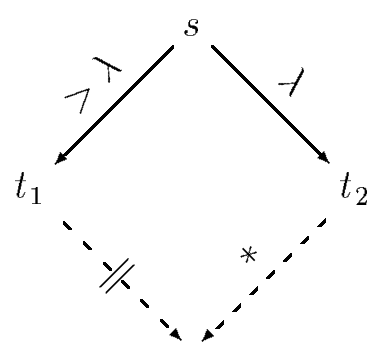

case (ii)

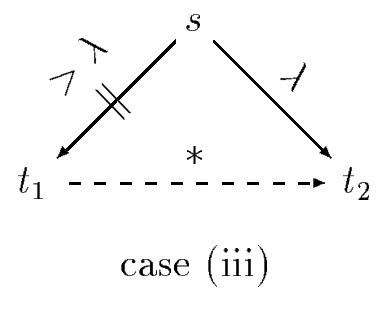

Figure 4: parallel critical pair condition PCPC

(i) If $P=\{\lambda\}$, i.e., $D$ is an outside critical peak, then there exists $t_{3}$ such that $t_{1}-\| t_{3} * t_{2} \cdot{ }^{9}$

(ii) If $P=\{p\}, p>\lambda$, i.e., $D$ is an inside (ordinary) critical peak, then there exists $t_{3}$ such that $t_{1} \longrightarrow t_{3} *_{\leftarrow} t_{2}$.

(iii) If $P=\left\{p_{1}, \ldots, p_{n}\right\}, n \geq 1$ such that $p_{i}>\lambda$ for some $i, 1 \leq i \leq n$, i.e., $D$ is an inside parallel critical peak, then $t_{1} \rightarrow^{*} t_{2}$.

The conditions (i)-(iii) of Definition 4.8 are depicted in Fig. 4.

\section{Lemma 4.9 (characterizing strong confluence of parallel reduction)}

For a given TRS, parallel reduction is strongly confluent if and only if for all terms $s, t_{1}$, $t_{2}$ we have: whenever $t_{1} \nVdash s \rightarrow t_{2}$, then there exists a term $t_{3}$ such that $t_{1} \rightarrow^{*} t_{3} \nVdash t_{2}$ (more succintly: $\operatorname{SCR}(\#) \Longleftrightarrow \# \circ \rightarrow \subseteq \rightarrow^{*} \circ \#$ ).

Proof: The direction ' $\Longrightarrow$ ' is obvious by definition of strong confluence and the facts

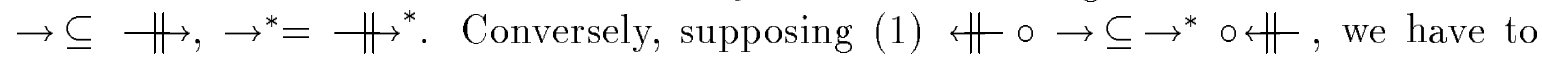

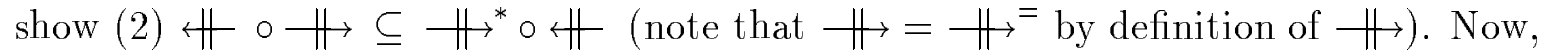
from (1) we obtain $\# \circ \rightarrow^{n} \subseteq \rightarrow^{*} \circ \#$ (for all $n \geq 0$ ) by a straightforward induction

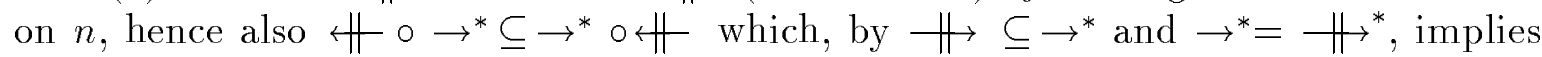
(2) as desired.

In fact, Lemma 4.9 can also be obtained as a special case from the following easy results about abstract reduction relations.

Lemma 4.10 (relating strong confluence of related abstract reduction relations)

Let $\rightarrow_{1}, \rightarrow_{2}$ be abstract reduction relations (on some set $A$ ). Then the following properties hold:

\footnotetext{
${ }^{9}$ Note again, that due to the asymmetry in the definition of (parallel) critical pairs / peaks this means that there must also exist a term $t_{4}$ with $t_{1} \rightarrow^{*} t_{4} \nVdash t_{2}$.
} 
(1) If ${ }_{1} \leftarrow \circ \rightarrow_{2} \subseteq \rightarrow_{2}^{*} \circ \overline{1}_{1} \leftarrow$ and $\rightarrow_{1} \subseteq \rightarrow_{2}^{*} \subseteq \rightarrow_{1}^{*}$, then $\operatorname{SCR}\left(\rightarrow_{1}\right)$.

(2) If $\operatorname{SCR}\left(\rightarrow_{1}\right)$ and $\rightarrow_{2} \subseteq \rightarrow_{1}^{*} \subseteq \rightarrow_{2}^{*}$, then $1 \leftarrow \circ \rightarrow_{2} \subseteq \rightarrow_{2}^{*} \circ \overline{\overline{1}} \leftarrow$.

Proof: Straightforward.

Taking $\rightarrow_{1}=\pitchfork$ and $\rightarrow_{2}=\rightarrow$, we obviously have $\rightarrow_{1}=\| \subseteq \rightarrow^{*}=\rightarrow_{2}^{*}=\rightarrow_{1}^{*}=\rightarrow^{*}$ and $\rightarrow_{2}=\rightarrow \subseteq \mathbb{H}^{*}=\rightarrow_{1}^{*}=\rightarrow_{2}^{*}=\rightarrow^{*}$. Hence, Lemma 4.9 follows by properties (1) and (2) of Lemma 4.10 above.

Now we are prepared for the main result of the paper.

\section{Theorem 4.11 (strong confluence of parallel reduction via parallel critical pairs)}

If a left-linear TRS $\mathcal{R}$ satisfies the parallel critical pair condition PCPC, then parallel reduction (in $\mathcal{R}$ ) is strongly confluent (more succinctly: $\operatorname{PCPC}(\mathcal{R}) \Longrightarrow \operatorname{SCR}(-\|)$ ), and hence, (ordinary reduction $\rightarrow$ in) $\mathcal{R}$ is also confluent.

Proof: Let $\mathcal{R}$ be a left-linear TRS satisfying $\operatorname{PCPC}(\mathcal{R})$. According to Lemma 4.9 it suffices to show that whenever we have a local divergence of the form

$$
D: t_{1} \nVdash s \rightarrow t_{2}
$$

there exists a term $t_{3}$ such that

$$
t_{1} \rightarrow^{*} t_{3} \# t_{2} .
$$

Hence, suppose $s$ reduces to $t_{1}$ by contracting $n$ parallel redexes $s / p_{1}, \ldots, s / p_{n}$ in $s$ using the rules $l_{1} \rightarrow r_{1}, \ldots, l_{n} \rightarrow r_{n}$ of $\mathcal{R}$ and substitutions $\sigma_{1}, \ldots, \sigma_{n}$, respectively. We may assume that the set $P=\left\{p_{1}, \ldots, p_{n}\right\}$ of parallel redex positions is non-empty, i.e., $n \geq 1$ (the case $n=0$ is trivial!). Further, suppose that $s$ reduces to $t_{2}$ by contracting some redex $s / q$ in $s$ using some rule $l \rightarrow r$ of $\mathcal{R}$ with substitution $\sigma$.

We proceed by a complete case distinction according to the relative positions of the contracted redexes (at $p_{1}, \ldots, p_{n}, q$ in $s$ ) in $D$ (this case analysis is illustrated in Fig. 5 below).

(1) $q \| P$ (parallel redexes): In this case we obviously get $t_{1} \rightarrow_{q} t_{3} P \nVdash t_{2}$ by commuting the steps, with $t_{3}$ defined by $t_{3}=t_{1}\left[q \leftarrow t_{2} / q\right]=t_{2}\left[p_{i} \leftarrow t_{1} / p_{i} \mid 1 \leq\right.$ $i \leq n]$.

(2) $\exists p_{i} \in P: q=p_{i}$ (overlay case): If the applied rules at $s / p_{i}=s / q$, i.e., $l_{i} \rightarrow r_{i}$ and $l \rightarrow r$, are the same, we are done since we then have: $t_{2} \|_{P \backslash\left\{p_{i}\right\}} t_{1}=: t_{3}$. Otherwise, the local divergence $t_{1} / q_{\lambda, l_{i} \rightarrow r_{i} \leftarrow s / q} \rightarrow_{q, l \rightarrow r} t_{2} / q$ (obtained from $D$ by considering only the subterm $s / q$ ) is an instance of a critical overlay (between the rules $l_{i} \rightarrow r_{i}$ and $l \rightarrow r$ ), hence by (the symmetric version of) the assumption $\operatorname{PCPC}(\mathcal{R})(\mathrm{i})$ we get $t_{1} / q \rightarrow^{*} t_{3}^{\prime} \# t_{2} / q$ for some $t_{3}^{\prime}$. Combining the reductions appropriately and exploiting commutation of parallel redex contractions we obtain the existence of $t_{1}^{\prime}$, $t_{3}$ with $s \rightarrow_{q} s^{\prime} \rightarrow_{\geq q}^{*} t_{1}^{\prime}, t_{2}\left\|_{\geq q} t_{1}^{\prime}-\right\|{ }_{P \backslash\{q\}} t_{3}$, $s \rightarrow_{q} s^{\prime} \|_{P \backslash\{q\}} t_{1} \rightarrow_{\geq q}^{*} t_{3}$. Hence, $t_{1} \rightarrow^{*} t_{3} \nVdash t_{2}$ as desired. 


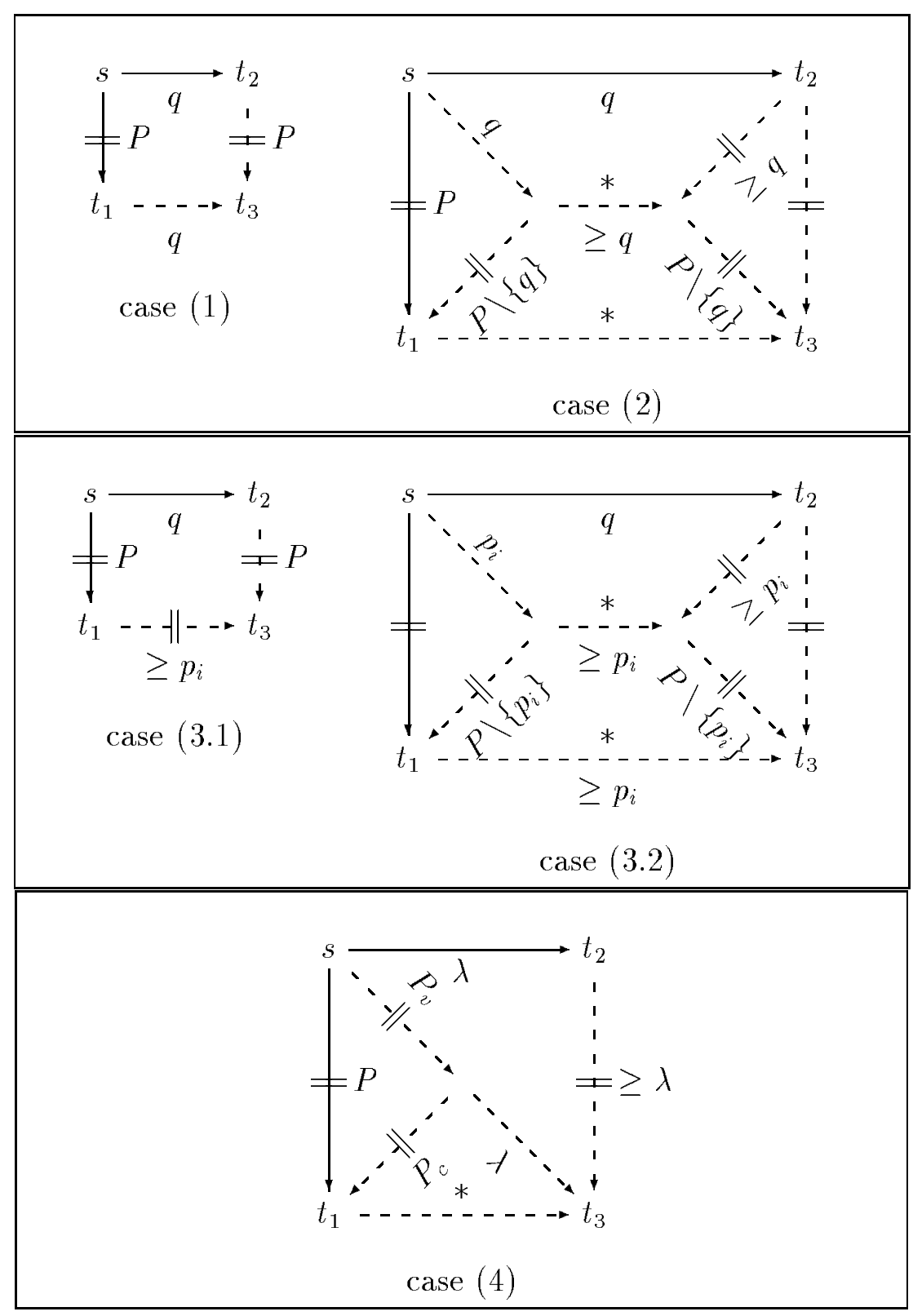

Figure 5: illustration of the cases of the proof of Theorem 4.11 
(3) $\exists p_{i} \in P: q>p_{i}$ (the single redex is below one of the parallel redexes): In this case we further distinguish between a variable and critical overlap.

(3.1) $\exists p \in \mathcal{V} \operatorname{Pos}\left(l_{i}\right): q \geq p_{i} p$ (variable overlap below $p_{i}$ ): Let $l_{i} / p=x \in \mathcal{V}$ and $p^{\prime}=q \backslash\left(p_{i} p\right)$. Define $\sigma_{i}^{\prime}$ by Dom $\sigma_{i}^{\prime}=\operatorname{Dom} \sigma, \sigma_{i}^{\prime} y=\sigma_{i} y$ for $y \neq x$, and $\sigma_{i}^{\prime} x=\sigma_{i} x\left[p^{\prime} \leftarrow \sigma r\right]$. Then we get $s=s\left[p_{i}=\sigma_{i} l_{i}\right] \longrightarrow{ }_{P} t_{1}=t_{1}\left[p_{i}=\right.$ $\left.\sigma_{i} r_{i}\right] \longrightarrow t_{1}\left[p_{i} \leftarrow \sigma_{i}^{\prime} r_{i}\right]=: t_{3}$ and $s \rightarrow_{q} t_{2} \prod_{P} t_{3}$ as desired.

(3.2) $q \backslash p \in \mathcal{F} \operatorname{Pos}\left(l_{i}\right)$ (critical overlap below $p_{i}$ ): Let $p^{\prime}=q \backslash p_{i}$. Then the mirrored version of the (extracted) divergence $t_{1} / p_{i}=\sigma_{i} r_{i} \lambda^{\leftarrow} \sigma_{i} l_{i}=s / p_{i}=$ $s / p_{i}\left[p^{\prime}=\sigma l\right] \rightarrow p^{\prime} s / p_{i}\left[p^{\prime} \leftarrow \sigma r\right]=t_{2} / p_{i}$ is an instance of an (ordinary) inside critical peak of $\mathcal{R}$ (obtained by overlapping $l \rightarrow r$ into $l_{i} \rightarrow r_{i}$ at position $p^{\prime}$ in $l_{i}$ ). By assumption (ii) of $\operatorname{PCPC}(\mathcal{R})$ we obtain $t_{1} / p_{i} \rightarrow_{\geq \lambda}^{*} t_{3}^{\prime}$ and $t_{2} / p_{i} \longrightarrow_{\geq \lambda} t_{3}^{\prime}$ for some $t_{3}^{\prime}$. Combining the reductions appropriately and exploiting commutation of parallel redex contractions we obtain the existence of $s^{\prime}, t_{2}^{\prime}, t_{3}$ with $s \rightarrow_{p_{i}} s^{\prime}\left\|_{P \backslash\left\{p_{i}\right\}} t_{1} \rightarrow_{\geq p_{i}}^{*} t_{3}, s \rightarrow_{q} t_{2}\right\|_{\geq p_{i}} t_{2}^{\prime} \|_{P \backslash\left\{p_{i}\right\}} t_{3}$, hence $t_{1} \rightarrow^{*} t_{3} \# t_{2}$ as desired.

(4) $\exists p_{i} \in P: q<p_{i}$ (some parallel redexes are below the single redex): In this case (which is the most difficult one) we can simplify the discussion a bit. Namely, if we succeed to close the extracted divergence $t_{1} / q P^{\prime} \| s / q \rightarrow_{\lambda} t_{2} / q$, where $P^{\prime}=$ $\left\{q \backslash p_{i} \mid p_{i} \geq q\right\}$, in the desired form, i.e., if $t_{1} / q \rightarrow_{\lambda}^{*} t_{3}^{\prime} \# t_{2} / q$ for some $t_{3}^{\prime}$, then we obtain $t_{1} \rightarrow_{\geq q}^{*} t_{3} \nVdash t_{2}$ from $s\left\|_{P_{1}} t_{1}^{\prime}\right\|_{P_{2}} t_{1} \rightarrow_{\geq q}^{*} t_{3}, s \rightarrow_{q} t_{2}\left\|_{\geq q} t_{2}^{\prime}\right\|_{P_{2}} t_{3}$ (where $t_{1}^{\prime}=s\left[p \leftarrow t_{1} / p \mid p \in P_{1}\right], t_{2}^{\prime}=t_{2}\left[q \leftarrow t_{3}^{\prime}\right], t_{3}=t_{1}\left[q \leftarrow t_{3}^{\prime}\right]=t_{2}\left[q \leftarrow t_{3}^{\prime}\right][p \leftarrow$ $\left.\left.t_{1} / p \mid p \in P_{2}\right], P_{1}=\left\{q p_{i}^{\prime} \mid p_{i}^{\prime} \in P^{\prime}\right\}, P_{2}=P \backslash P_{1}\right)$, simply by commutation of independent steps and by combining twice two independent parallel steps into one parallel step. Hence, w.l.o.g. we may assume $q=\lambda$ and $\forall p \in P: p>\lambda$, i.e., $D$ has the form $t_{1} P \| s \rightarrow_{\lambda} t_{2}$. The idea now is to treat the 'variable overlap part' of the parallel step first, and then tackle the remaining 'parallel peak' by using assumption (iii) of $\operatorname{PCPC}(\mathcal{R})$. So, let $P_{v}=\left\{p \in P \mid \exists p^{\prime} \in \mathcal{V} \operatorname{Pos}(l): p \geq p^{\prime}\right\}$, $P_{c}=\{p \in P \mid p \in \mathcal{F} \operatorname{Pos}(l)\}$. Clearly, we have $P=P_{v} \uplus P_{c}$. Now, due to leftlinearity of $\mathcal{R}$ we obtain (as in case (3.1) above) $s=\sigma l-\|_{P_{v}} s^{\prime}=\sigma^{\prime} l \rightarrow_{\lambda} \sigma^{\prime} r$, $s=\sigma l \rightarrow_{\lambda} \sigma r=t_{2}-\sigma^{\prime} r$, for some $\sigma^{\prime}$. Furthermore, by definition of $P_{v}, P_{c}$, we get $s^{\prime}=\sigma^{\prime} l-{ }_{P_{c}} t_{1}$. Since all redex positions from $P_{c}$ in $s^{\prime}$ are critical (w.r.t. the step $\sigma^{\prime} l \rightarrow_{\lambda, l \rightarrow r} \sigma^{\prime} r$ ), the divergence $t_{1} P_{c} \nVdash s^{\prime}=\sigma^{\prime} l \rightarrow_{\lambda} \sigma^{\prime} r$ must be an instance of an inside parallel critical peak of $\mathcal{R}$, according to Lemma 4.7. Assumption (iii) of $\mathrm{PCPC}(\mathcal{R})$ yields joinability from left to right, i.e., $t_{1} \rightarrow^{*} \sigma^{\prime} r$. Defining now $t_{3}:=\sigma^{\prime} r$ we are done, since we have obtained $t_{1} \rightarrow^{*} t_{3} \nVdash t_{2}$ as desired.

Since non-overlapping TRSs have no critical pairs, hence also no parallel critical pairs, we obtain as a direct consequence of Theorem 4.11 the following well-known result.

Corollary 4.12 (cf. e.g. [Ros73])

Any orthogonal TRS is confluent. 
For readers which are familiar with the proof of Huet/Toyama for Theorem 3.7 we remark that one crucial difference between their proof (of Theorem 3.7) and ours (of Theorem 4.11 ) is the following. In the case that the considered local divergence $t_{1} \nVdash s \rightarrow t_{2}$ has the form $t_{1} P \mathbb{H} s \rightarrow_{\lambda, \sigma, l \rightarrow r} t_{2}$ where at least one of the positions $p \in P$ is critical w.r.t. the outside step, i.e., $p \in \mathcal{F} \operatorname{Pos}(l)$, the proof of Huet/Toyama appeals to the induction hypothesis, whereas we proceed as described, namely by exploiting case (iii) of the parallel critical pair condition $\operatorname{PCPC}(\mathcal{R})$. Interestingly, these two approaches seem to be incompatible, as the examples below suggest.

\section{Discussion and Related Work}

We give (non-terminating) examples showing that the presented confluence criteria SCCP, $\mathrm{CPC}_{\mathrm{HT}}$ and PCPC are not comparable with each other. In fact, for the former two this is well-known.

Example 5.1 (Example 3.8 continued, left-linear case: PCPC $\nRightarrow \mathrm{CPC}_{\mathrm{HT}}$ ) In this example it is easily verified that $\operatorname{PCPC}(\mathcal{R})$ is indeed satisfied. In particular, for the (proper) parallel critical peak $f\left(a^{\prime}, a^{\prime}\right) \nVdash f(a, a) \rightarrow_{\lambda} b$ we have $f\left(a^{\prime}, a^{\prime}\right) \rightarrow b$, hence $f\left(a^{\prime}, a^{\prime}\right) \rightarrow^{*} b$ as desired. However, $\mathrm{CPC}_{\mathrm{HT}}$ is not applicable, since e.g. the inside critical peak $f\left(a^{\prime}, a\right) \leftarrow f(a, a) \rightarrow_{\lambda} b$ is not parallel closed, i.e., we do not have $f\left(a^{\prime}, a\right) \nVdash b$. Moreover, the strongly closed criterion SCCP is also not applicable, because $\mathcal{R}$ is not right-linear.

Example 5.2 (left- and right-linear case: PCPC $\nRightarrow \mathrm{CPC}_{\mathrm{HT}}, \mathrm{SCCP}$ ) The TRS

$$
\mathcal{R}=\left\{\begin{aligned}
f(a) & \rightarrow f(g(b, b)) \\
a & \rightarrow g(c, c) \\
c & \rightarrow d \\
d & \rightarrow b \\
b & \rightarrow d
\end{aligned}\right.
$$

is left- and right-linear (and non-terminating). Moreover, it is easily checked that $\mathcal{R}$ satisfies $\operatorname{PCPC}(\mathcal{R})$, but neither $\mathrm{CPC}_{\mathrm{HT}}(\mathcal{R})$ nor $\operatorname{SCCP}(\mathcal{R})$.

Example 5.3 (left- and right-linear case: SCCP $\nRightarrow$ PCPC) The TRS

$$
\mathcal{R}=\left\{\begin{aligned}
f(a, b) & \rightarrow c \\
a & \rightarrow a^{\prime} \\
b & \rightarrow b^{\prime} \\
c & \rightarrow f\left(a^{\prime}, b\right) \\
c & \rightarrow f\left(a, b^{\prime}\right) \\
c & \rightarrow f(a, b)
\end{aligned}\right.
$$

is left- and right-linear (and non-terminating). Furthermore, as it is easy to check, we have $\operatorname{SCCP}(\mathcal{R})$ but neither $\mathrm{CPC}_{\mathrm{HT}}(\mathcal{R})$ nor $\operatorname{PCPC}(\mathcal{R})$. This means that $\mathcal{R}$ is strongly confluent. Note, however, that parallel reduction is only confluent here, but not strongly confluent. 


\section{Example 5.4 (left-linear case: $\mathrm{CPC}_{\mathrm{HT}} \nRightarrow \mathrm{PCPC}$ )}

The TRS

$$
\mathcal{R}=\left\{\begin{aligned}
f(a, a) & \rightarrow g(f(a, a)) \\
a & \rightarrow b \\
f(b, x) & \rightarrow g(f(x, x)) \\
f(x, b) & \rightarrow g(f(x, x))
\end{aligned}\right.
$$

is left-linear (and non-terminating). Furthermore, it is obviously parallel closed, i.e., $\mathrm{CPC}_{\mathrm{HT}}(\mathcal{R})$ holds. However, it doesn't satisfy the parallel critical pair condition $\operatorname{PCPC}(\mathcal{R})$ (because the proper parallel critical pair $\langle f(b, b), g(f(a, a))\rangle$ is not joinable from left to right, i.e., $f(b, b) \rightarrow^{*} g(f(a, a))$ does not hold).

The last example shows in particular, that $\mathrm{CPC}_{\mathrm{HT}}$ is not subsumed in general by PCPC. However, there is one special case where $\mathrm{CPC}_{\mathrm{HT}}$ is indeed (properly) subsumed by PCPC, namely when there are no proper parallel critical pairs (but only ordinary inside ones). In that case, parallel closedness of all inside critical pairs implies conditions (ii) and (iii) of PCPC, according to $\mathrm{CPC}_{\mathrm{HT}}$ (ii). This is obvious for PCPC(ii), and PCPC(iii) only has to hold for (ordinary) inside critical peaks which is guaranteed again by $\mathrm{CPC}_{\mathrm{HT}}(\mathrm{ii})$.

Finally, let us briefly discuss related work and possible extensions of our approach. First of all, it seems rather straightforward to generalize our approach to a 'commutation setting' analogous to the one of Toyama ([Toy88]) mentioned above for Theorem 3.7.

Furthermore, we would like to mention one possible source of refinements for Theorem 4.11 (and related critical pair based confluence criteria) which is based on making use of the ambiguity of rewriting. More precisely, it may be the case that a parallel critical peak $t_{1}>\lambda \nVdash s \rightarrow_{\lambda} t_{2}$ as in PCPC(iii) is not joinable from left to right as required. However, it might e.g. be (an instance of) another outside critical peak $t_{1}^{\prime} \lambda^{\leftarrow} s^{\prime} \rightarrow_{\lambda} t_{2}^{\prime}$ which satisfies PCPC(i), i.e., $t_{1}^{\prime} \nVdash t_{3}{ }^{*} \leftarrow t_{2}^{\prime}$ and $t_{1}^{\prime} \rightarrow^{*} t_{4} \nVdash t_{2}^{\prime}$ for some $t_{3}^{\prime}, t_{4}^{\prime}$. In this case the reasoning in the proof of Theorem 4.11 still applies. The following simple example ilustrates this kind of refinement.

\section{Example 5.5 (refining PCPC)}

The TRS

$$
\mathcal{R}=\left\{\begin{aligned}
f(a) & \rightarrow f(f(a)) \\
a & \rightarrow b \\
f(x) & \rightarrow f(b)
\end{aligned}\right.
$$

does not satisfy PCPC(iii) since for the inside critical peak $f(b)>\lambda_{\lambda} f(a) \rightarrow_{\lambda} f(f(a))$ we do not have $f(b) \rightarrow^{*} f(f(a))$. However, we also have the outside critical peak $f(b) \lambda^{\leftarrow} f(a) \rightarrow_{\lambda} f(f(a))$, which is strongly joinable in the sense of PCPC(i), due to $f(f(a)) \rightarrow f(b)$. Hence, parallel reduction in $\mathcal{R}$ is strongly confluent.

Similar refinements are also possible for Theorem 3.7.

Another direction of extending our approach is to combine it with the recent results of van Oostrom ([Oos94b]) who showed that the approach of Huet/Toyama can be generalized by replacing parallel reduction steps by the more general so-called (complete) 
developments, using essentially the same proof structure as in Theorem $3.7 .^{10}$ The basic idea of developments roughly is that a set of rewrite steps is considered to be parallel if a 'parallel extraction' of all the steps in the set is possible ([Oos94b]). This implies for instance, that in a (left-linear) TRS a derivation $s=\sigma l \rightarrow_{p} \sigma^{\prime} l \rightarrow_{\lambda, \sigma^{\prime}, l \rightarrow r} \sigma^{\prime} r$ (with $p \geq q$ for some $q \in \mathcal{V} \operatorname{Pos}(l)$ ) can be viewed as a single parallel step. It seems that our approach via parallel critical pairs developed in this paper and the approach of van Oostrom generalizing the notion of being 'parallel' are 'orthogonal' to each other, hence it should be possible to combine both as well as the corresponding proof structures.

Similarly, it is conceivable that our approach can also be combined with (proof techniques for) confluence results for the higher-order rewrite systems of [MN94].

Last but not least we think that the notion of parallel critical pairs that we have explicitly introduced here might turn out to be fruitful for other purposes, too. But this remains to be seen.

Acknowledgements: I thank Vincent van Oostrom and Claus-Peter Wirth for useful comments and Paul Taylor for his diagrams.

Final Note: It should be mentioned that Yoshihito Toyama, after having read (a longer version of) this paper, informed the author ([Toy95]) about the existence of another very early paper, namely "Y. Toyama: On the Church-Rosser property of term rewriting systems, NTT ECL Technical Report 17672 (Dec. 23, 1981), in Japanese”, containing very similar ideas and results. The relationships between the latter paper and ours as well as possible extensions and refinements will be investigated in joint future research.

\section{References}

[AM90] J. Avenhaus and K. Madlener. Term rewriting and equational reasoning. In R.B. Banerji, editor, Formal Techniques in Artificial Intelligence. A Sourecbook, volume 6 of Studies in Computer Science and Artificial Intelligence. North-Holland, 1990.

[BD88] L. Bachmair and N. Dershowitz. Critical pair criteria for completion. Journal of Symbolic Computation, 6(1):1-18, 1988.

[BS94] F. Baader and J.H. Siekmann. Unification theory. In D.M. Gabbay, C.J. Hogger, and J.A. Robinson, editors, Handbook of Logic in Artificial Intelligence and Logic Programming, volume 2 of Handbooks of Logic in Computer Science and of Logic in Artificial Intelligence and Logic Programming, pages 41-126. Clarendon Press, Oxford, 1994.

[DJ90] N. Dershowitz and J.-P. Jouannaud. Rewrite systems. In J. van Leeuwen, editor, Formal models and semantics, Handbook of Theoretical Computer

\footnotetext{
${ }^{10}$ Actually, the corresponding result in [Oos94b] is even more general, since it also holds for (certain) left-linear higher-order systems, called patterm rewriting systems there.
} 
Science, volume B, chapter 6, pages 243-320. Elsevier - The MIT Press, 1990.

[DJK91] N. Dershowitz, J.P. Jouannaud, and J.W. Klop. Open problems in rewriting. In R.V. Book, editor, Proc. 4th Int. Conf. on Rewriting Techniques and Applications, volume 488, pages 445-456. Springer-Verlag, 1991.

[Hue80] G. Huet. Confluent reductions: Abstract properties and applications to term rewriting systems. Journal of the ACM, 27(4):797-821, oct 1980.

[KB70] D.E. Knuth and P.B. Bendix. Simple word problems in universal algebra. In J. Leech, editor, Computational Problems in Abstract Algebra, pages 263297. Pergamon Press, Oxford, U. K., 1970. Reprinted 1983 in "Automation of Reasoning 2", Springer, Berlin, pp. 342-376.

[Klo92] J.W. Klop. Term rewriting systems. In S. Abramsky, D. Gabbay, and T. Maibaum, editors, Handbook of Logic in Computer Science, volume 2, chapter 1, pages 2-117. Clarendon Press, Oxford, 1992.

[KMN88] D. Kapur, D.R. Musser, and P. Narendran. Only prime superpositions need be considered in the Knuth-Bendix completion procedure. Journal of Symbolic Computation, 1988(6):19-36, 1988.

[Küc85] W. Küchlin. A confluence criterion based on the generalized Newman lemma. In B. Caviness, editor, Proc. EUROCAL'85, volume 204 of Lecture Notes in Computer Science, pages 390-399. Springer-Verlag, 1985.

[MN94] Richard Mayr and Tobias Nipkow. Higher-order rewrite systems and their confluence. Technical Report TUM-I9433, TU München, August 1994.

[New42] M.H.A. Newman. On theories with a combinatorial definition of equivalence. Annals of Mathematics, 43(2):223-242, 1942.

[Oos94a] V. v. Oostrom. Confluence by decreasing diagrams. Theoretical Computer Science, 121:259-280, May 1994.

[Oos94b] V. v. Oostrom. Developing develpments. Technical Report ISRL-94-4, Basic Research Laboratories, NTT, Japan, December 1994.

[OT94] M. Oyamaguchi and Y. Toyama. On the Church-Rosser property of Eoverlapping and simple-right-linear TRS's. Technical Report COMP94-29 (1994-07), IEICE, 1994.

[Pla94] D.A. Plaisted. Equational reasoning and term rewriting systems. In D.M. Gabbay, C.J. Hogger, and J.A. Robinson, editors, Handbook of Logic in Artificial Intelligence and Logic Programming - Logical Foundations, volume 1 of Handbooks of Logic in Computer Science and of Logic in Artificial Intelligence and Logic Programming, pages 273-364. Clarendon Press, Oxford, 1994. 
[Ros73] B.K. Rosen. Tree-manipulating systems and Church-Rosser theorems. Journal of the ACM, 20:160-187, 1973.

[TO95] Y. Toyama and M. Oyamaguchi. Church-Rosser property and unique normal form property of non-duplicating term rewriting systems. In N. Dershowitz and N. Lindenstrauss, editors, Proc. of 4th Int. Workshop on Conditional (and Typed) Term Rewriting Systems, Jerusalem, Israel (1994), volume 968 of Lecture Notes in Computer Science, pages 316-331. Springer-Verlag, 1995.

[Toy88] Y. Toyama. Commutativity of term rewriting systems. In K. Fuchi and L. Kott, editors, Programming of Future Generation Computer, volume II, pages 393-407. North-Holland, 1988.

[Toy95] Y. Toyama. Personal e-mail communication, August 31, 1995.

[WB86] F. Winkler and B. Buchberger. A criterion for eliminating unnecessary reductions in the Knuth-Bendix algorithm. In J. Demetrovics, G. Katona, and A. Salomaa, editors, Proc. Colloquium on Algebra, Combinatorics and Logic in Computer Science, Györ, Hungary, 1983, volume II of Colloquia Mathematica Societatis János Bolyai, 42, pages 849-869. North Holland, 1986. 